\title{
岷江上游林线附近岷江冷杉种群的生存分析
}

\author{
程 伟 吴 宁* 罗 鹏 \\ (中国科学院成都生物研究所, 成都 610041)
}

\begin{abstract}
摘 要 岷江冷杉 (Abies faxoniana) 林是青藏高原东南缘亚高山暗针叶林的主要类型之一, 在岷江上游地区, 岷江 冷杉林一般在 $2800 \sim 3800 \mathrm{~m}$ 之间呈带状分布并且在阴坡形成林线。为了对岷江上游林线地段的岷江冷杉种群进 行生存分析, 在峮江上游林线附近弓杜岭 $\left(33^{\circ} 02^{\prime} 39^{\prime \prime} \mathrm{N}, 103^{\circ} 43^{\prime} 11^{\prime \prime} \mathrm{E}\right)$ 设置了 10 个样地, 使用侧生轮枝计数或 WinDENDRO 系统对个体年龄进行确定。调查数据经匀滑技术处理, 编制了峮江冷杉种群特定时间生命表, 绘制了 峮江冷杉种群的存活曲线、死亡率曲线和消失率曲线。结果表明: 1) 峮江上游林线地段岷江冷杉种群存活曲线趋 于 Deevey- III型。2)林线地段峮江冷杉种群生存率呈单调下降趋势, 生存率下降趋势前期高于后期, 说明怅江冷杉 种群幼苗死亡率高, 种群后期比较稳定。3)林线地段峎江冷杉林整个生长期中出现了两个死亡高峰期, 一个出现 在幼苗向幼树过渡时期 ( $0 \sim 40$ 年), 另一个出现在中龄时期 (180 年)。岷江上游林线地段岷江冷杉种群幼苗死亡 率较高的原因可能和林线地段的温度较低、积雪厚度、风向、水分等环境条件有关。
\end{abstract}

关键词 峮江冷杉种群 林线 特定时间生命表 存活曲线 生存分析

\section{SURVIVAL ANALYSIS OF ABIES FAXONIANA POPULATIONS NEAR TIMBERLINE ON THE UPPER MINJIANG RIVER}

\author{
CHENG Wei WU Ning ${ }^{*}$ and LUO Peng \\ ( Chengdu Institute of Biology, Chinese Academy of Sciences, Chengdu 610041, China)
}

\begin{abstract}
Abies faxoniana forests are one of the major types of sub-alpine dark coniferous forests on the eastern Tibetan Plateau. In the mountainous areas of the upper Minjiang River, it is distributed between altitudes of $2800-3800 \mathrm{~m}$ a.s.l and usually forms the upper timberline on shaded slopes. To explore the survival status of $A$. faxoniana populations, ten plots $(20 \mathrm{~m} \times 20 \mathrm{~m})$ were investigated and analyzed in Gonggangling $\left(33^{\circ} 02^{\prime} 39^{\prime \prime} \mathrm{N}, 103^{\circ} 43^{\prime} 11^{\prime \prime} \mathrm{E}\right)$. Individual ages were determined using WinDENDRO system or by counting tree branches. Time-specific life tables or vertical life table curves of survival-mortality based and hazard based age structures were drawn. The results showed that: 1) The survival curve of the population conformed to the type of Deevey- III ; 2) With an increase in the population mortality ratio and a decrease in the survival rate, the mortality ratio greatly increased in ages $0-40$ years, reaching $89.7 \%$; 3) There existed two peaks of mortality in the lifespan, one was from seedlings to young trees $(0-40$ years $)$ and the other in the adult stage ( 180 years). It is suggested that the mortality rate of $A$. faxoniana seedlings is impacted most by temperature, wind, snowpack depth, water and other environmental factors.
\end{abstract}

Key words Population of Abies faxoniana, Timberline, Time-specific life table, Survival curve, Spectral analysis

种群统计 (Demography) 是研究种群数量动态的 一种方法, 它的核心是建立种群全部生活史的各年 龄组成、生活态级出生率及死亡率, 甚至包括迁移率 在内的信息综合表, 即生命表 (Life table) (周纪纶 等, 1992; 谢宗强等, 1999; 间淑君等, 2002a, 2002b)。 特定时间生命表( Time-specific life table) 是在某一特
定时间断面上依据种群内部多个龄级的存活状况编 成, 其多应用于长寿种群, 特别是乔木树种的生命表 的编制。

岷江冷杉 ( Abies faxoniana) 林是青藏高原东南缘 亚高山暗针叶林的主要类型之一, 也是四川特有的 森林类型, 主要分布于青藏高原东南缘以及四川盆

基金项目: 中国科学院重大项目 (KSCX1-07)、重要方向项目（KSCX2-01-09）、国家“十五”攻关项目（2001BA606A-05）以及四川省青年科技基 金项目 (03ZQ026-043) 
地西缘山地 (四川植被协作组, 1980; 杨玉坡和李承 彪, 1992)。岷江冷杉是青藏高原东南缘形成林线的 主要树种之一, 在其分布的核心区域一一岷江上游 地区, 岷江冷杉常常在 $3000 \mathrm{~m}$ 以上区域以纯林形 式存在, 为该地区阴坡林线的主要建群树种, 是区域 森林生态屏障的主体, 但人为干扰和破坏也比较严 重。近年来, 有关高山林线的成因问题已成为气候 变化生态学效应以及高山植被格局变化研究的热点 之一。本文旨在通过对林线附近岷江冷杉进行生存 分析, 为进一步探讨青藏高原东南缘现状林线成因 及森林格局变化的机理提供参考(吴宁, 1998)。

\section{1 研究区域概况}

研究区域位于青藏高原东南缘岷山山脉东段的 弓杜岭, 是长江重要支流峮江的发源地, 地理位置约 为 $33^{\circ} 02^{\prime} 39^{\prime \prime} \mathrm{N}, 103^{\circ} 43^{\prime} 11^{\prime \prime} \mathrm{E}$, 主峰海拔高 $3858 \mathrm{~m}$ 。 该地带的年平均气温为 $2.8{ }^{\circ} \mathrm{C}, 1$ 月均温 $-7.6{ }^{\circ} \mathrm{C}$, 7 月均温 $9.7{ }^{\circ} \mathrm{C}$, 无绝对无霜期, 年 $\geqslant 10{ }^{\circ} \mathrm{C}$ 积温 $428.6{ }^{\circ} \mathrm{C}$; 年平均降水量 $717.7 \mathrm{~mm}$, 其中 $80 \%$ 集中 在 5 10 月。研究地点位于林线以下的亚高山段, 紫外辐射强, 气温变化大, 昼夜温差大, 土壤主要是 山地暗棕壤, 土层较厚, 地表调落物较多(陈文年等, 2003)。

岷江冷杉在本地区分布以沟谷阴坡和半阴坡湿 润环境为主, 其下部与紫果云杉 ( Picea purpurea) 群 落相接, 在植被垂直带谱中, 共同组成亚高山暗针叶 林的主体, 其分布上至 $3700 \mathrm{~m}$ 处形成林线 (部分地 段可沿沟谷上升到 $3800 \mathrm{~m}$ )。在弓杜岭的林线附近 峮江冷杉基本以纯林形式存在, 部分地点由于砍伐 和自然形成的林窗有阔叶树种侵入, 并可形成一定 的郁闭度。

\section{2 研究方法}

\section{1 样地设置及取样}

在对岷江冷杉林的整个分布区做全面勘查的基 础上, 以岷江上游弓杜岭阴坡林线及附烬地段为主 要调查地点, 在海拔 $3500 \sim 3700 \mathrm{~m}$ 地段采用相邻 格子法设置典型样地 10 个, 每个样地面积为 $20 \mathrm{~m} \times$ $20 \mathrm{~m}$, 记录每个样地的海拔、坡向、坡度等指标, 对样 地内进行每木调查, 调查样地内所有树木的胸径、树 高和冠幅等数据(张利权, 1990; 刘智慧, 1990; 刘峰 等, 2000)。对灌木层采用 $5 \mathrm{~m} \times 5 \mathrm{~m}$ 、草本层采用 $1 \mathrm{~m}$ $\times 1 \mathrm{~m}$ 样方进行调查。同时记录样地内外的植物生 长情况。

\section{2 年龄确定及结构分析}

由于岷江冷杉种群生长周期长, 不可能追踪所 有个体的生长周期, 因此只有通过研究现实不同年 龄阶段的个体数量统计来推断种群时间上的动态过 程。对于 20 龄以下的岷江冷杉采取数侧生轮枝的 方法, 确定其年龄。对于侧生轮枝不明显的 20 龄以 下和 20 龄以上的峮江冷杉个体, 则使用生长雉在胸 径处 $(1.3 \mathrm{~m})$ 取样, 每木取 3 个样品, 带回实验室使 用 WinDENDRO 年轮分析系统 (Made in Régent Instrument INC, Canada) 确定其年龄, 并建立相关的年龄胸径回归方程。对于病腐树木及所谓空心树, 则测 量出其胸径, 用年龄-胸径回归方程估算其年龄。

\section{3 结果与分析}

\section{1 生命表的编制}

由于所研究峮江冷杉为天然林, 而且所收集的 数据是同一时期岷江冷杉种群所有个体的年龄数 据, 它反映了多个世代重叠的年龄动态历程的一个 特定时间, 而不是对同生种群的全部生活史的追踪 (冯士雍, 1983), 因此在生命表的编制中会出现死亡 率为负值的情况, 对于这种现象的出现, 有人认为 （江洪, 1983)“生命表分析中产生的一些负的 $D$ 值 $X$ 值, 这与数学假设不符, 但仍能提供有用的生态学记 录, 即表明种群处于迅速发展或衰落之中”。因此本 文采用匀滑技术处理数据, 经匀滑修正得 $a_{x}$, 再据 此编制出岷江冷杉的特定时间生命表(表 1)（毕晓 丽等, 2001)。

\section{2 存活曲线、死亡率曲线及消失率曲线}

以岷江冷杉的特定时间生命表为基础, 以标准 化存活数 $l_{x}$ 为纵坐标, 以龄级中值为横坐标绘制存 活曲线 (图 1), 以 $q_{x}, K_{x}$ 为纵坐标, 以龄级中值为横 坐标绘制死亡率曲线和消失率曲线 (图 2)。

根据 1947 年 Deevey (江洪, 1983) 的划分, 存活 曲线可以分为 3 种类型: Deevey- I 型, Deevey- II 型, Deevey- III 型曲线。从图 1 来看, 弓杜岭冷杉种群的 存活曲线更接近于 Deevey- III型。

岷江冷杉的存活曲线表明前期的存活数呈直线 下降趋势, 环境篮的选择强度大, 大约只有 $10 \%$ 的 幼苗能通过此篎进入下一龄级,一旦进入下一龄级, 幼树生长趋势趋于稳定, 动态变化趋于平衡。

如图 2 所示, 岷江冷杉的死亡率曲线和消失率 曲线大致重合, 变化趋势基本一致。死亡率和消失 率都出现两个高峰期。第一个高峰期出现在 $0 \sim 40$ 年, 幼苗死亡率高达 $90 \%$, 其原因可能与岷江冷杉 
表 1 岷江冷杉种群特定时间生命表

Table 1 The time specific life table of Abies faxoniana

\begin{tabular}{cccccccccccc}
\hline$x$ & $\Delta_{x}$ & $\begin{array}{c}\text { 实际数目 } \\
\text { Actual number }\end{array}$ & $a_{x}$ & $l_{x}$ & $d_{x}$ & $q_{x}$ & $L_{x}$ & $T_{x}$ & $e_{x}$ & $S_{x}$ \\
\hline 20 & 40 & 369 & 369 & 1000 & 897.02 & 0.900 & 551.49 & 909.21 & 0.91 & 0.10 & 2.27 \\
60 & 40 & 38 & 38 & 102.98 & 2.71 & 0.026 & 101.63 & 357.72 & 3.47 & 0.97 & 0.03 \\
100 & 40 & 13 & 37 & 100.27 & 18.97 & 0.190 & 90.79 & 256.10 & 2.55 & 0.81 & 0.21 \\
140 & 40 & 15 & 30 & 81.30 & 18.97 & 0.230 & 71.82 & 165.31 & 2.03 & 0.77 & 0.27 \\
180 & 40 & 23 & 23 & 62.33 & 29.81 & 0.480 & 47.43 & 93.50 & 1.50 & 0.52 & 0.66 \\
220 & 40 & 12 & 12 & 32.52 & 8.13 & 0.250 & 28.46 & 46.07 & 1.42 & 0.75 & 0.29 \\
260 & 40 & 53 & 9 & 24.39 & 18.97 & 0.780 & 14.91 & 17.62 & 0.72 & 0.22 & 1.50 \\
300 & 40 & 35 & 2 & 5.42 & 5.42 & 1 & 2.71 & 2.71 & 0.50 & 0 \\
\hline
\end{tabular}
$a_{x}$ : 在 $x$ 龄级内现存的个体数 $l_{x}$ : 在 $x$ 龄级开始时标准化存活个体数, $l_{x}=a_{x} / a_{0} \times 1000 d_{x}$ : 从 $x$ 到 $x+1$ 间隔期内标准化死亡数 $q_{x}$ : 从 $x$ 到 $x+1$ 龄级间隔期间个体死亡率 $q_{x}=d_{x} / l_{x} \quad L_{x}$ : 从 $x$ 到 $x+1$ 龄级间隔期间平均存活的个体数, $L_{x}=\left(l_{x}+l_{x+1}\right) / 2 \quad T_{x}$ : 从 $x$ 龄级到超过 $x$ 龄级的存活的个体总数, $T_{x}=\sum L_{x} \quad e_{x}$ : 进入 $x$ 龄级个体的生命期望寿命, $e_{x}=T_{x} / l_{x} \quad S_{x}$ : 存活率, 即 $x+1$ 期存活个体数与 $x$ 期存活个体数之 比, $S_{x}=l_{x+1} / l_{x} \quad K_{x}$ : 致死力或消失率, 即从 $x$ 到 $x+1$ 器受到的阻力, $K_{x}=\ln \left(l_{x}\right)-\ln \left(l_{x+1}\right)$ 。 $x$ : Midpoint age x (in years) $\Delta_{x}$ : Width of age (in years) $\quad a_{x}$ : Number of alive organisms at age $x \quad l_{x}$ : Proportion organisms surviving from the beginning of the life table to age $x, l_{x}=a_{x} / a_{0} \times 1000 \quad d_{x}$ : Number of dead organisms from age $x$ to $x+1, d_{x}=a_{x}-a_{x+1} \quad q_{x}$ : Mortality rate from age $x$ to $x+1, q_{x}=d_{x} / l_{x} \quad L_{x}$ : Number of year of lived by the total of the cohort 100000 births in the interval $x, x+1 \quad L_{x}=\left(l_{x}+l_{x+1}\right) / 2 \quad T_{x}$ : Total number of alive organisms from age $x ; T_{x}=\sum L_{x} \quad e_{x}:$ Life expectancy at age $x, e_{x}=T_{x} / l_{x} \quad S_{x}$ : Age-specific survival, $S_{x}=l_{x+1} / l_{x} \quad K_{x}$ : Age-specific mortality, $K_{x}=\ln \left(l_{x}\right)-\ln \left(l_{x+1}\right)$

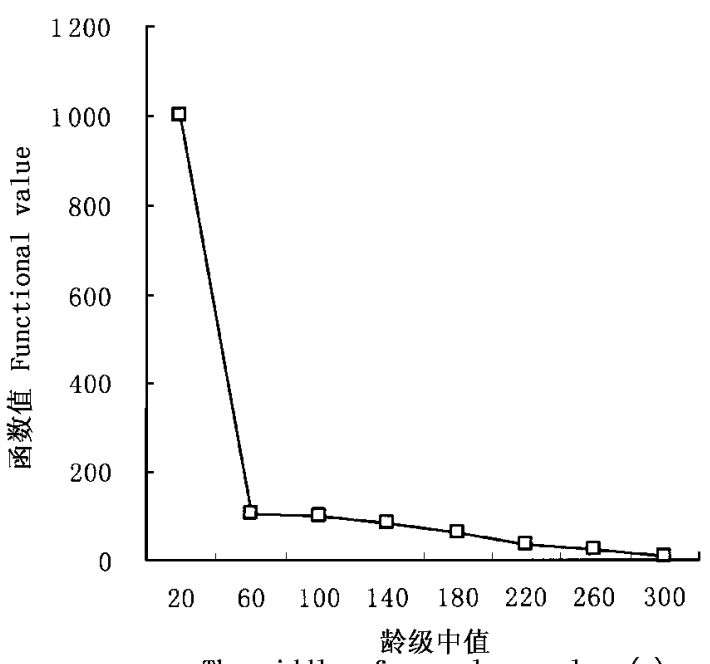

图 1 岷江冷杉种群存活曲线

Fig. 1 Survival curve of Abies faxoniana population

林的更新模式和所处位置有关。岷江冷杉天然林更 新模式主要为林窗更新, 幼苗生长空间相对狭小, 幼 苗成活率与灌从盖度、温度、湿度以及光照条件等有 关。另外, 弓杜岭的岷江冷杉林分布于林线地段, 气 候条件恶劣, 导致幼苗成活率极度降低。第二个死 亡高峰期处于 180 年附近, 推测可能是该阶段岷江 冷杉处于青壮年期, 个体对营养空间的需求不断增 大, 林内光照、水分和空间等因子不能充分满足其需 求, 植株间竞争激烈, 自疏和他疏作用增强, 个体差 异显著, 导致第二个死亡高峰期出现。

平均生命期望 $\left(e_{x}\right)$ 反映 $\mathrm{x}$ 龄级内个体的生命期
望寿命。岷江冷杉的生命期望表明岷江冷杉幼苗一 旦长成树, 则有高的生命期望寿命。

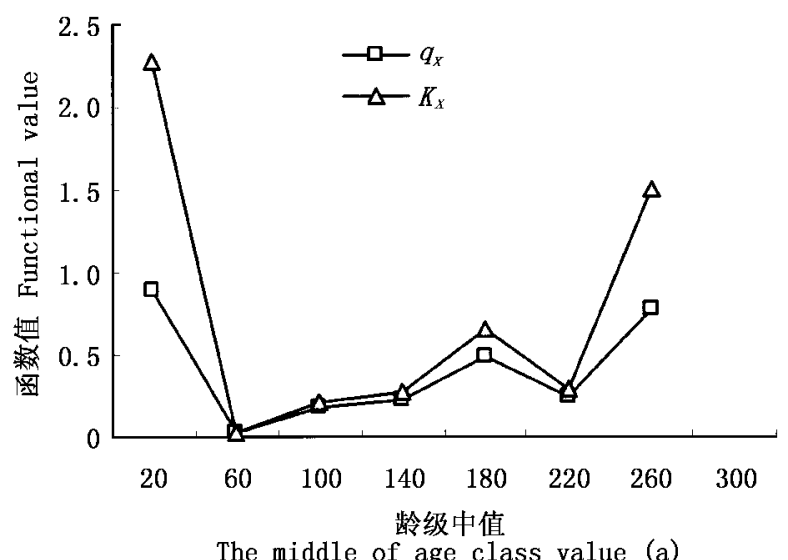

图 2 岷江冷杉种群死亡率 $\left(q_{x}\right)$ 及消失率 $\left(K_{x}\right)$ 曲线

Fig.2 Mortality $\left(q_{x}\right)$ and hazard rate $\left(K_{x}\right)$ of Abies faxoniana population

\section{4 讨 论}

岷江上游林线附近岷江冷杉种群生存分析表 明, 岷江冷杉种群死亡最高峰出现在幼苗阶段, 岷江 冷杉的存活曲线接近 Deevey- III 型。岷江冷杉幼苗 死亡率高的原因可能与其生物学特性及环境因素有 关。本文研究的林线地段海拔位于 $3500 \sim 3700 \mathrm{~m}$ 附近, 相对于林线下方地段, 树木已经接近其生长上 限, 树木的成活率可能与环境因子在海拔梯度上的 变化有关 (Hoch et al.,2002)。在温带地区林线地段 夏季最热月温度一般在 $10{ }^{\circ} \mathrm{C}$ 左右 $($ Körner \& 
Larcher, 1988; Körner, 1998), 低于此温度树木则由 于热量亏损而导致不能生存, 相对于光合作用来说, 生长对温度更敏感。在岷江上游林线地段, 温度低 于植物生长所要求的最低温度, 导致植物形成新的 细胞及组织逐渐变慢甚至不可能, 意味着温度对幼 树有更大的限制作用, 造成林线地段幼树死亡率较 高。Häteenschwiler 和 Smith(1999)研究表明, 林线地 段风向、积雪厚度也是决定树苗成活率的关键因素: 在迎风向, 由于风的原因, 积雪很少, 导致幼苗几乎 不能存活。在下风向, 积雪厚度在 $0.5 \sim 1.5 \mathrm{~m}$ 之间 对幼苗生长有利, 太多的积雪导致生长季节的缩短 和碳平衡的不足; 太少的积雪则导致生长季节和繁 殖期水分的不足。冬季寒冷造成的干燥也可能是导 致树木存活率降低的原因, 特别是冬末或初春季节, 由于气温回暖可促使树木地上部分提前萌动生长, 而根部由于还处在冻土中, 则会导致树木生理性的 干旱, 这种损害对幼树的影响更加明显 (Marchand \& Chabot, 1978; 吴宁和刘照光, 1998)。在弓杜岭的林 线附近, 冻土往往要到 5 月底才完全解冻, 而这时的 日均温已上升到 $7 \sim 8{ }^{\circ} \mathrm{C}$, 因此这种影响是显而易见 的。此外可能还有一些次要因素也导致了幼苗成活 率降低, 如在林线附近树木受到的机械性损伤很难 被修复, 植物体内糖和氨基酸的合成过程不能达到 组织更新所需要的最小速率 (James et al ., 1994 ; Benecke \& Havranek, 1980)。总的来说, 岷江冷杉种群 幼树对于林线区域环境条件的耐受性远远低于成年 树, 死亡率较高的原因是林线地段温度较低、风向、 积雪厚度、冬季干燥导致干旱缺水等因素综合作用 的结果。

以前的静态生命表多采用“空间序列”代替“时 间序列” 的基本思路, 即以径级代替龄级进行生命表 分析。在林线区域, 由于大量树木畸形生长及矮曲 林的出现, 这种径级代替龄级的方法影响了分析结 果的准确性, 在此情况下, 对用径级来代表龄级的办 法应采取谨慎的态度。本文使用 WinDENDRO 年轮 分析系统, 结合生长锥取样法确定岷江冷杉的种群 寿命, 既避免了使用解析木对林木本身的破坏, 又最 大限度地保证了数据的准确性。

\section{参 考 文 献}

Benecke U, Havranek WM (1980). Gas-exchange of trees at altitudes up to timberline. In: Benecke U, Davis MR eds. Mountain Environments and Subalpine Tree Growth. Forest Research Institute Technical, New Zealand, 70, $195-212$.

Bi XL (毕晓丽), Hong W (洪伟), Wu CZ (吴承祯), Yan SJ
(闻淑君)（2001）. Life table analysis of Castanopsis carlesh population in Wuyishan. Journal of Tropical and Subtropical Botany (热带亚热带植物学报), 9, 243-247. (in Chinese with English abstract)

Chen WN (陈文年), Wu N (吴宁), Luo P (罗鹏), Yan ZL (晏 兆莉) (2003). Species diversity and arbor population distribution pattern of Sabina przewalskii community in the forest-grassland ecotone in the watershed of upper Minjiang river. Chinese Journal of Applied and Environmental Biology (应用与环境生物学报), 9, 221 - 225. (in Chinese with English abstract)

Collaborating Group for Vegetation of Sichuan (四川植被协作组) (1980). Vegetation of Sichuan (四川植被). Sichuan People's Press, Chengdu, 133-187. (in Chinese)

Feng SY (冯士雍) (1983). Survival analysis III. Mathematics in Practice and Theory (数学的实践与认识), 1, 70-76. (in Chinese with English abstract)

Hoch G, Popp M, Körner C (2002). Altitudinal increase of mobile carbon pools in Pinus cembra suggests in limitation growth at the Swiss treeline. Oikos, 98,361-374.

James JC, Grace J , Hoad SP (1994). Growth and photosynthesis of Pinus sylvestris at its altitudinal in Scotland. Joural of Ecology, 82, $297-306$.

Jiang H (江洪) (1983). Population Ecology of Spruce (云杉种群 生态学). Science Press, Beijing, 11 13. (in Chinese)

Körner C (1998) . A re-assessment of high elevation treeline positions and their explanation. Oecologia, 115,445-459.

Körner C, Larcher W (1988). Plant life in cold climates. Plant and Temperature, $42,25-57$.

Liu F (刘峰), Chen WL (陈伟烈), He JS (贺金生) (2000). Population structure and regeneration of Quercus aliena var. acuteserrata in Shennongjia. Acta Phytoecologica Sinica (植物生 态学报), 24, 396-401. (in Chinese with English abstract)

Liu ZH (刘智慧) (1990). A primary study on the structure and dynamics of Castanopsis fargesii on Jinyun mountain, Sichuan Province. Acta Phytoecologica et Geobotanica Sinica (植物生态 学与地植物学学报), 14,120-127. (in Chinese with English abstract)

Marchand PJ, Chabot B(1978). Winter water relations of tree-line plant species on Mt. Washington. Arctic and Alpine Research, 10, $105-116$.

Häteenschwiler S, Smith WK (1999). Seedling occurrence in alpine conifers: a case study from the Rocky Mountains, USA. Acta Oecologica, 20,219-224.

Wu N (吴宁) (1998). The ecological characteristics of Abies fabric population in Gongga Mountain. In: Chen FB( 陈富斌), Luo J (罗辑) eds. The Ecological Studies on Alpine Environment of Gongga Mountain (贡嘎山高山生态环境研究). Vol.2. China Meteorological Press, Beijing, 16-22. ( in Chinese with English abstract)

Wu N (吴宁), Liu ZG (刘照光) (1998). Probing into the causes of geographical pattern of subalpine vegetation on the eastern 
Qinghai-Tibetan Plateau. Chinese Journal of Applied and Environmental Biology (应用与环境生物学报), 3, 290-297. (in Chinese with English abstract)

Xie ZQ (谢宗强), Chen WL (陈伟烈), Lu P (路鹏), Hu D (胡东) (1999). The demography and age structure of the endangered plant population of Cathaya argyrophylla. Acta Ecologica Sinica (生态学报), 19, 523-528. (in Chinese with English abstract)

Yan SJ (间淑君), Hong W (洪伟), Wu CZ (吴承桢), Bi XL (毕晓丽) (2002a). Life process and spectral analysis of Castanopsis fargesii population. Chinese Journal of Applied and Environmental Biology (应用与环境生物学报) , 8,351-355. (in Chinese with English abstract)

Yan SJ (间淑君), Hong W (洪伟), Wu CZ (吴承桢), Bi XL (毕晓丽), Lan B (蓝斌) ( 2002b). The structure and distribu- tion pattern of dominant population in Castanopsis carlesii community. Journal of Tropical and Subtropical Botany (热带亚热带 植物学报), 10, 15-21. (in Chinese with English abstract)

Yang YP(杨玉坡)，Li CB (李承彪) (1992). Forest in Sichuan (四川森林). China Forestry Publishing House, Beijing, $196-$ 218. (in Chinese)

Zhou JL (周纪纶), ZHeng SZ (郑师章), Yang C (杨持) (1992). Population Ecology of Botany (植物种群生态学). Higher Education Press, Beijing, 45 - 125. (in Chinese)

Zhang LQ (张利权) (1990). The age structure and spatial pattern of population of Pinus taiwanensis in Songyang county, Zhejiang Province. Acta Phytoecologica et Geobotanica Sinica (植物生态 学与地植物学学报), 14, 328 - 334. (in Chinese with English abstract)

责任编委: 党承林 责任编辑: 张丽赫

\section{“第三届现代生态学讲座”会议通知}

近些年来, 生态学在国际范围内获得迅速发展, 它的应用范围越来越广。为了交流现代生态学的理论、方法与发展趋势, 探讨中国生态学今后的发展方向, 北京师范大学和中国科学院植物所在国家自然基金委员会支持下, 决定于 2005 年 6 月 $1 \sim 7$ 日在北京师范大学组织召开 “现代生态学讲座 (III) 暨学术讨论会”, 组织国内外相关学科和领域的科学家对世界生态学的发展 开展开放式的学术交流和综合性的学术讨论。通过这次讲座, 拟提出 10 个专题报告(分子与进化生态学、生理生态学、种群与 群落生态学、生态系统生态学、景观生态学、全球变化及其生态响应、生物多样性与生态系统功能、保护生物学、恢复生态学以 及生态健康、生态评价和生态服务), 内容涉及多学科、多领域、多层次, 对现代生态学研究的热点问题加以综述, 以期反映现 代生态学的新理论、新观点、新方法及国际上生态学的热点。该讲座的目的是纵观国际生态学发展趋势和进展, 展望与制定 中国生态学发展战略和生态学科重点 (优先) 发展领域(纲领), 凝炼今后中国在生态学科上的重大科学问题, 为国家生态环境 与经济建设提供更为有效的服务。

\section{会议秘书处}

负责人: 葛剑平 010-58808999, gejp@bnu.edu.cn 白永飞 010-82595771, yfbai@ ibcas.ac.cn

联络人: 王天明 010-58805291, moderneco@126.com 王红芳 010-58805291, wanghf6.6@163.com 